\title{
Long Non-Coding RNA NEAT1 Regulates Pyroptosis in Diabetic Nephropathy via Mediating the miR-34c/NLRP3 Axis
}

\author{
Jin-Feng Zhan ${ }^{a}$ Hong-Wei Huang ${ }^{a}$ Chong Huang ${ }^{b}$ Li-Li Hu ${ }^{b}$ Wen-Wei Xu ${ }^{c}$ \\ a Medical Examination Center, the Second Affiliated Hospital of Nanchang University, \\ Nanchang, PR China; ${ }^{b}$ Department of Nephrology, the Second Affiliated Hospital of Nanchang \\ University, Nanchang, PR China; 'Institute of Clinical Pharmacology, Nanchang University, \\ Nanchang, PR China
}

\section{Keywords}

NEAT1 $\cdot$ miR-34c $\cdot$ NLRP3 $\cdot$ Pyroptosis $\cdot$ Inflammation

\begin{abstract}
Introduction: Diabetic nephropathy (DN) is a serious complication of diabetes mellitus and is considered to be a sterile inflammatory disease. Increasing evidence suggest that pyroptosis and subsequent inflammatory response play a key role in the pathogenesis of DN. However, the underlying cellular and molecular mechanisms responsible for pyroptosis in DN are largely unknown. Methods: The rat models of DN were successfully established by single 65 $\mathrm{mg} / \mathrm{kg}$ streptozotocin treatment. Glomerular mesangial cells were exposed to $30 \mathrm{mmol} / \mathrm{L} \mathrm{high}$ glucose media for $48 \mathrm{~h}$ to mimic the DN environment in vitro. Gene and protein expressions were determined by quantitative real-time PCR and Western blot. Cell viability and pyroptosis were measured by MTT assay and flow cytometry analysis, respectively. The relationship between IncRNA NEAT1, miR-34c, and Nod-like receptor protein-3 (NLRP3) was confirmed by luciferase reporter assay. Results: We found that upregulation of NEAT1 was associated with the increase of pyroptosis in DN models. miR-34c, as a target gene of NEAT1, mediated the effect of NEAT1 on pyroptosis in DN by regulating the expression of NLRP3 as well as the expressions of caspase- 1 and interleukin-1 $\beta$. Either miR-34c inhibition or NLRP3 overexpression could reverse the accentuation of pyroptosis and inflammation by sh-NEAT1 transfection in the in vitro model of DN. Conclusions: Our findings suggested NEAT1 and its target gene miR-34c regulated cell pyroptosis via mediating NLRP3 in DN, providing new insights into understanding the molecular mechanisms of pyroptosis in the pathogenesis of DN.
\end{abstract}




\section{Kidney \\ Blood Pressure \\ Research}

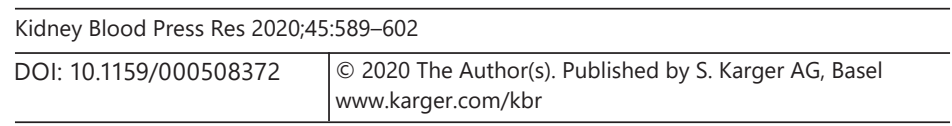

Zhan et al.: IncRNA NEAT1/miR-34c/NLRP3 Axis Regulates Pyroptosis in DN

\section{Introduction}

Diabetic nephropathy (DN) is one of the serious microvascular complications of diabetes mellitus and is the leading cause of end-stage renal disease worldwide as a result of the growing population with diabetes. The pathology of DN is characterized by proliferation, extracellular matrix accumulation and fibrosis, as well as podocyte depletion [1]. However, the aetiology of this disease is multifactorial, and the underlying mechanisms have not been fully elucidated due to the complex pathogenesis of DN including inflammation, epithelial to mesenchymal transition (EMT), oxidative stress, mitochondrial damage, apoptosis, and genetic/epigenetic factors [2]. Among those, chronic low-grade renal inflammation has been well documented by experimental and clinical studies to play a crucial role in promoting the development and progression of DN [3]. A number of molecules related to the inflammation pathways are therefore considered to be the molecular targets for the treatment of DN. However, recent evidence suggested that some novel pathways, such as pyroptosis, also represent important pathogenic mechanisms in DN [4,5]. Various states caused by diabetes, such as inflammation, hyperglycaemia, prolonged cellular stress, and mitochondrial impairment, contribute to the process of cell death in different types of cells in DN [6]. Different from apoptosis, pyroptosis is an inflammatory form of programmed cell death, mediated by caspase- 1 activation following Nod-like receptor protein-3 (NLRP3) inflammasome complex formation resulting in cell lysis and pro-inflammatory cytokine release [7]. The inflammatory response caused by pyroptosis is therefore proposed to be a contributor to the chronic inflammation presented in DN. However, the underlying cellular and molecular mechanisms responsible for pyroptosis in DN have not been fully elucidated.

Long non-coding RNAs (lncRNAs) are a heterogeneous type of transcript with over 200 nucleotides, which have low or no protein coding potentials. It is evident that lncRNAs regulate gene expression on different levels and serve a wide range of biological functions in development and diseases [8]. Notably, recentstudies suggest a close relationship between IncRNAs and DN. A number of lncRNAs have been identified to contribute to the pathogenesis and progression of DN [9]. For example, IncRNA TUG1 has been reported to alleviate extracellular matrix accumulation via downregulating microRNA-377 (miR-377) in a mouse model of DN [10] and regulate the mitochondria function in podocytes [11]. IncRNA-NR_033515 promoted mesangial cell proliferation and increased the expression of proliferation-related and fibrogenesis-related genes by regulating miR-743b-5p expression in DN patients [12]. Most recent studies also suggested that lncRNA-Neat1 (NEAT1) exerted a key role in DN, demonstrating that the expression of NEAT1 was upregulated in both in vivo and in vitro models of DN [13]. A further study also demonstrated that NEAT1 accelerated proliferation and EMT, contributing to fibrogenesis in DN [14]. Recently, Zhang et al. [15] revealed that NEAT1 promoted the activation of inflammasome and caspase-1, and enhanced cytokine production and pyroptotic cell death in mouse macrophages. However, the role of NEAT1 in DN-related pyroptosis has not been investigated.

Different from IncRNAs, miRNAs are short non-coding RNAs containing about 22 nucleotides. Both miRNA and IncRNA contain structural domains that can bind other RNAs via complementary base pairing. This RNA-RNA interaction impacts on gene expression and modulates fundamental cellular process, and contributes to the pathogenesis of many diseases, including DN. Indeed, emerging evidence from observations of animal models and specific cell lines suggest the involvement of miRNAs in the pathogenesis of DN [16]. Zhao et al. [17] found that miR-30c was downregulated in a $d b / d b$ mouse model of DN while overexpression of miR-30c protects against DN by suppressing EMT and fibrogenesis in both $d b / d b$ mice and high glucose (HG)-treated human kidney cells. Guo et al. [18] expounded that upregulated miR-29c increased the expression of inflammatory cytokines and promoted the 


\section{Kidney \\ Blood Pressure \\ Research}

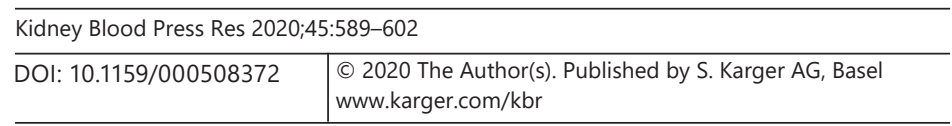

Zhan et al.: IncRNA NEAT1/miR-34c/NLRP3 Axis Regulates Pyroptosis in DN

progress of DN in DN patients. A meta-analysis on the miRNA expression profile in DN revealed downreglulation of miR-34c in at least two studies [19]. Recent studies observed that miR-34c inhibited HG-induced cell death in podocytes [20] and suppressed inflammasome activity in astrocytes, microglia, and neurons [21]. However, a potential role of miR-34c in regulating pyroptosis in DN is unclear.

In the present study, we aimed to determine the role of NEAT1 and miR-34c in DN pathogenesis with respect to pyroptosis and inflammation as well as the underlying mechanism in a rat model of DN and a mesangial cell line. This work provides new insights and solid evidence for understanding the initiation and progression of DN, as well as providing ideas for developing new prevention and treatment measures.

\section{Materials and Methods}

\section{Animals}

This animal study was performed in accordance with the guidelines by the National Institutes of Health Guide for the Care and Use of Laboratory Animals. All protocols were approved by the Animal Ethics Committee of the Second Affiliated Hospital of Nanchang University. The rat models of streptozotocin (STZ)-induced DN were established as previously described [22]. Male Sprague-Dawley rats $(n=10)$ weighting $200 \pm 20 \mathrm{~g}$ at 8 weeks old were housed under standard conditions of a controlled temperature at $22-24^{\circ} \mathrm{C}$ and humidity at $40-70 \%$ with a 12-h light/dark cycle. Animals had free access to standard chow and water. After at least 5 days of acclimatization to the environment, rats were randomized to receive intraperitoneal administration of a single dose of $65 \mathrm{mg} / \mathrm{kg} \mathrm{STZ} \mathrm{(dissolved} \mathrm{in} 0.1 \mathrm{~mol} / \mathrm{L}$ citric acid buffer with pH 4.5; Sigma, St Louis, MO, USA) or vehicle after overnight fasting (at least $12 \mathrm{~h}$ ). The blood glucose level was measured by a blood glucose meter (FreeStyle Libre; Abbott, Alameda, CA, USA) after 3 days of STZ/vehicle injection, and rats with fasting blood glucose level $>16.7 \mathrm{mmol} / \mathrm{L}$ were considered as successful DN models. Rats were then euthanized under halothane anaesthesia. Both kidneys were removed from 5 STZ-treated rats and 5 vehicle-treated rats under sterile conditions, and the renal cortex was sliced into 3-mm masses. Glomeruli were isolated using a sequential sieving method as previously described [23]. Glomeruli were isolated from both kidneys of each rat. Five kidney tissues from 5 rats underwent RNA extraction and subsequent quantitative real-time PCR (qPCR) analysis; the remaining five kidney tissues were used to extract total protein for subsequent Western blot analysis.

\section{Cell Culture and Treatment}

The rat glomerular mesangial cell line (HBZY-1) was obtained from the American Type Culture Collection (ATCC, Manassas, VA, USA) and maintained at $37^{\circ} \mathrm{C}, 5 \% \mathrm{CO}_{2}$ in DMEM (Gibco, Carlsbad, CA, USA) supplemented with $10 \%$ FBS. The full length of sequences of NEAT1 were amplified and inserted into pcDNA3.1 vector (Life Technologies, San Francisco, CA, USA). The eukaryotic vector expressing ghrelin was termed as pcDNA-NEAT1, and the empty vector served as control. By using the same method, NLRP3 was cloned and termed as pcDNA-NLRP3. Short-hairpin (sh) RNAs directed against NEAT1, miR-34c mimics, mimics control (mimics NC), miR-34c inhibitor, and its negative control (inhibitor NC) were synthesized by GenePharma (Shanghai, China). Cells were transfected using Lipofectamine 3000 (Life Technologies). The medium was replaced with fresh culture medium $6 \mathrm{~h}$ after transfection, and mesangial cells were harvested for subsequent studies at $48 \mathrm{~h}$ after transfection. Cells were then exposed to $30 \mathrm{mmol} / \mathrm{L} \mathrm{HG}$ media for $48 \mathrm{~h}$ to mimic the DN environment in vitro [24]. All experiments were performed in triplicate and repeated at least twice. 


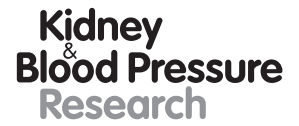

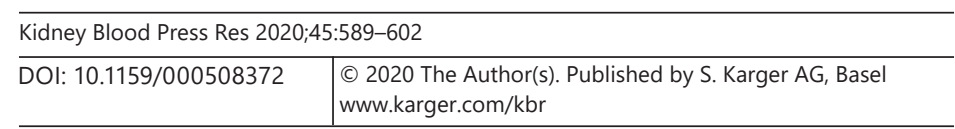

Zhan et al.: IncRNA NEAT1/miR-34c/NLRP3 Axis Regulates Pyroptosis in DN

\section{MTT Assay}

The viability of HBZY1 cells was determined using a 3-(4,5-dimethylthiazol-2-yl)-2,5-diphenyltetrazolium bromide (MTT) cell proliferation assay as previously described [25]. In brief, when cell density had reached $80 \%$ following the transfection and treatment, HBZY1 cells were seeded in a 96-well plate. After 24,48 , and $72 \mathrm{~h}$ of incubation, the cells were then incubated with a medium containing $10 \%$ MTT solution (Sigma). After incubation for an additional $4 \mathrm{~h}$, the supernatant was aspirated and $100 \mu \mathrm{L}$ of DMSO (Sigma) was added to each well and gently shaken for $10 \mathrm{~min}$. When the formazan crystals were completely dissolved, the optical density (OD) values at $570 \mathrm{~nm}$ were measured using a microplate reader (DeTech Experimental Equipment, Nanjing, China). Each experiment was repeated 3 times. With the OD value as the ordinate, and cell viability curve was plotted.

\section{Flow Cytometry}

Cell pyroptosis was measured using a FAM fluorescent-labelled inhibitor of caspase-1 assay (FLICA) and propidium iodide according to the manufacturer's instructions (Bio-Rad, Hercules, CA, USA). Pyrototic changes were detected by flow cytometry analysis. The collected data were analysed using FlowJo (FlowJo LLC, Ashland, OR, USA).

\section{Quantitative Real-Time PCR}

Total RNA was extracted from cells or mixed tissues of five kidneys from 5 rats using Trizol (Life Technologies). The quantity and integrity of RNA were determined using a NanoDrop spectrophotometer (Thermo Scientific, Waltham, MA, USA) and an Agilent Bioanalyzer RNA 6000 Nano kit (Agilent Technologies, Beijing, China), respectively. In addition, the measured OD260/OD280 of RNA is between 1.8-2.0; RNA electrophoresis results showed it included three distinct bands (28S, 18S, and 5S), and the brightness of $28 \mathrm{~S}$ is twice that of $18 \mathrm{~S}$. One microgram of RNA was reverse transcribed to first-strand cDNA using a HiFi Script cDNA synthesis kit (Life Technologies). The reverse-transcription step was carried out in duplicate to ensure accuracy. qPCR analysis was carried out using an Ultra SYBR Mixture kit according to the manufacturer's instructions (Fisher Scientific, USA) on an Applied Biosystems 7500 qPCR System (Life Technologies). PCR amplification efficiency was established by means of calibration curves. The cDNA template was continuously diluted 4 times by multiple of 10 to use in separate real-time reactions, and their threshold cycle values were determined. The amplification efficiencies ranged from 95 to $98 \%$. The primers used were as follows: NEAT1 (forward: TGGCTAGCTCAGGGCTTCAG, reverse: TCTCCTTGCCAAGCTTCCTTC); miR-34c (forward: GCTGCTGTAGGCAGTGTAGTTAG, reverse: CTCAACTGGTGTCGTGGAGTC); NLRP3 (forward: GTAGGTGTGGAAGCAGGACT, reverse: CTTGCTGACTGAGGACCTGA); caspase-1 (forward: TCCAGTATGACTCTACCCACG, reverse: CACGACATACTCAGCACCAG); interleukin (IL)-1 $\beta$ (forward: TCCAGTATGACTCTACCCACG, reverse: CACGACATACTCAGCACCAG); GAPDH (forward: CCATCAACGACCCCTTCATT, reverse: GACCAGCTTCCCATTCTCAG); and $\beta$-actin (forward: AGCGGGAAATCGTGCGTGAC, reverse: TCCASTGCCCAGGAAGGAAGG). The PCR reaction procedure: $95^{\circ} \mathrm{C}$ for $10 \mathrm{~min}$, followed by $95^{\circ} \mathrm{C}$ for $15 \mathrm{~s}, 60^{\circ} \mathrm{C}$ for $60 \mathrm{~s}, 72^{\circ} \mathrm{C}$ for $45 \mathrm{~s}$, for a total of 40 cycles, and finally $72^{\circ} \mathrm{C}$ for $10 \mathrm{~min}$. GAPDH and $\beta$-actin served as internal references according to the MIQE guidelines [26]. The relative expression level was calculated using the relative quantification $2^{-} \Delta \Delta^{\mathrm{Ct}}$ method to determine fold-change [27]. All samples were run in triplicate.

\section{Western Blotting}

Whole cells and kidney glomeruli from five kidney tissues of 5 rats were mixed and extracted using the radio-immunoprecipitation assay buffer in the presence of protease inhibitors (Roche, Mannheim, Germany) at $4^{\circ} \mathrm{C}$. Protein concentrations were measured using 


\section{Kidney \\ Blood Pressure \\ Research}

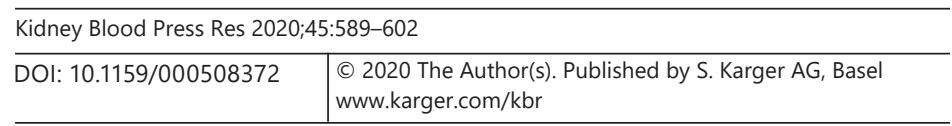

Zhan et al.: IncRNA NEAT1/miR-34c/NLRP3 Axis Regulates Pyroptosis in DN

a Direct Detect Spectrometer (Merck, Darmstadt, Germany); $30 \mu \mathrm{g}$ of proteins were electrophoresed by $15 \%$ SDS-PAGE as reducing conditions and then transferred onto PVDF membranes (Millipore, Bedford, MA, USA). After blocking with 5\% non-fat milk in TBST for 1 $\mathrm{h}$, the membranes were probed with the diluted primary rabbit antibodies against NLRP3 (ab214185, dilution 1:500; Abcam, Shanghai, China), caspase-1 (ab1872, dilution 1:1,000; Abcam), IL-1 (ab9722, dilution 1:1,000; Abcam), and GAPDH (ab9485, dilution 1:2,000; Abcam) overnight at $4{ }^{\circ} \mathrm{C}$. Then, the membranes were incubated with horseradish peroxidase-conjugated secondary anti-rabbit antibodies (ab97051, dilution 1:5,000; Abcam) for $2 \mathrm{~h}$ at room temperature and visualized using horseradish peroxidase-conjugated secondary antibody with enhanced chemiluminescence substrate on a BioRad Chemidoc MP system (Bio-Rad). ImageJ software was used to quantify the immunoblots. The fold-changes of the target proteins were normalized to that of GAPDH. All Western blots were repeated 3 times and means of normalized densities were calculated.

\section{Luciferase Reporter Assay}

The target sequence from NEAT1 or NLRP3 3'-untranslated region (3'-UTR) containing the predicted miR-34c binding site was cloned into pmirGLO Dual-Luciferase vector (Promega, San Luis Obispo CA, USA) to construct the reporter vector NEAT1 wild type (NEAT1 WT) and NLRP3 wild type (NLRP3 WT). Site mutations were carried out on the putative binding site of NEAT1 or NLRP3 in 3'-UTR, and the subsequent fragment was cloned to generate the NEAT1 mutated type (NEAT1 MUT) and the NLRP3 mutated type (NLRP3 MUT). Then the wild-type vector or the mutated vector and miR-34c mimics or mimics NC were co-transfected into cells. After $48 \mathrm{~h}$ of transfection, luciferase activity was measured using a Dual Luciferase Reporter Assay System (Promega).

\section{Statistical Analysis}

All normally distributed data are expressed as means \pm standard deviation (SD) of at least three independent experiments. Data were compared using Student's $t$ test or one-way ANOVA with post hoc analysis (Bonferroni test). Analysis was conducted using IBM SPSS Statistics 23 (IBM, Armonk NY, USA). A $p$ value of $<0.05$ was accepted as statistically significant.

\section{Results}

Upregulation of NEAT1 Expression and Increased Levels of Pyroptosis-Related Proteins in STZ-Induced DN Rats

In order to clarify the role of NEAT1 as well as its relationship with pyroptosis in DN, we firstly examined the expression of NEAT1 and pyroptosis-related proteins in an in vivo rat model. DN rat models were established using the STZ method. All 5 rats treated with STZ met the criteria with fasting blood glucose $>16.7 \mathrm{mmol} / \mathrm{L}$ and were classified as DN rats. Compared to the vehicle-treated rats, the gene expression of NEAT1 in the kidney was upregulated in the STZ-induced DN rats $(1.010 \pm 0.116$ vs. $1.426 \pm 0.128, p<0.05)$ (Fig. $1 \mathrm{~A})$. Western blotting also revealed that the protein levels of NLRP3 inflammasome, caspase-1, and pro-inflammatory cytokine IL-1 $\beta$ in the kidney was significantly increased in DN rats compared to the vehicle-treated rats $(0.330 \pm 0.053$ vs. $0.837 \pm 0.027, p<0.01 ; 0.363 \pm 0.026$ vs. $0.580 \pm 0.056$, $p<0.05$; $0.373 \pm 0.046$ vs. $0.537 \pm 0.035, p<0.05$; respectively) (Fig. $1 \mathrm{~B}$ ). These results indicate that cell pyroptosis is implicated in the pathogenesis of DN while upregulated expression of NEAT1 can be associated with the increased expressions of pyroptosis-related proteins. 


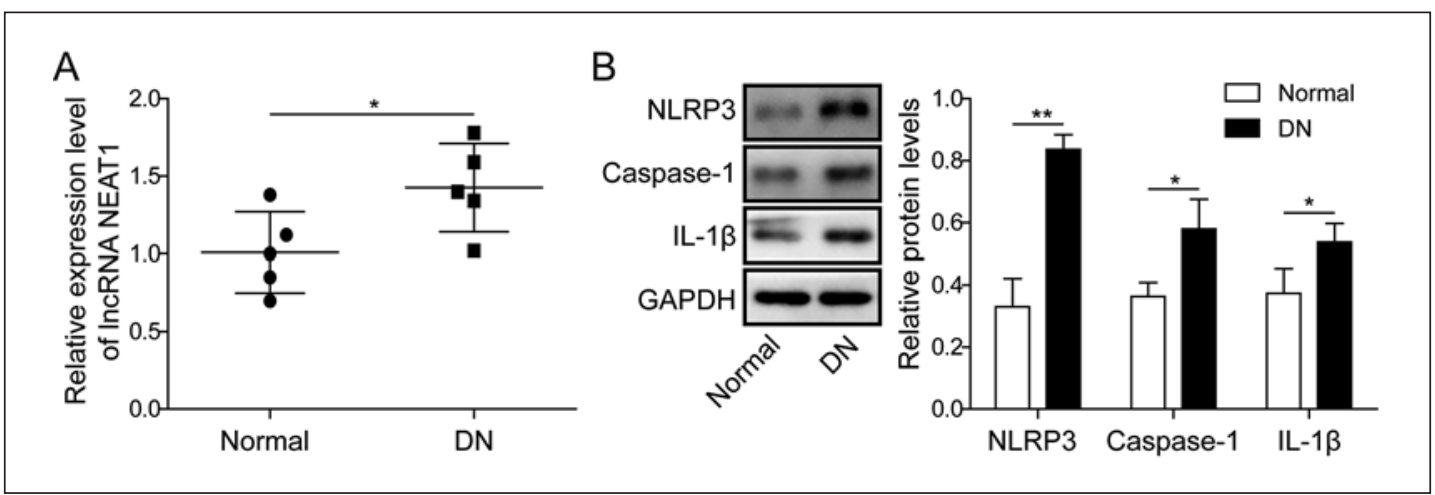

Fig. 1. Upregulation of NEAT1 expression and increased levels of pyroptosis-related proteins in STZ-induced DN rats. Male Sprague-Dawley rats were treated with STZ to establish a rat model of DN. All 5 rats treated met the criteria with fasting blood glucose $>16.7 \mathrm{mmol} / \mathrm{L}$. Kidney tissues from each vehicle-treated rat and STZ-treated rat were used for the following analyses. A qPCR: NEAT1 expression in the kidney. B Western blot with representative immunoblots of the corresponding proteins: the protein expression level of NLRP3, caspase- 1 , and IL-1 $\beta$ in the kidney. Data are expressed as means \pm SD. Comparisons were conducted using Student's $t$ test. $* p<0.05 ;{ }^{* *} p<0.01$.

\section{NEAT1 Promotes Cell Pyroptosis in HG-Treated HBZY1 Cells}

We explored the relationship between NEAT1 and pyroptosis in an in vitro DN model of HG-treated HBZY1 cells. Consistent with the results from the in vivo DN model, the expression of NEAT1 was upregulated in HG-treated HBZY1 cells compared with cells maintained in standard media $(0.993 \pm 0.081$ vs. $1.530 \pm 0.128, p<0.05)$ (Fig. $2 \mathrm{~A})$. Cells were then transfected with pcDNA-NEAT1 or sh-NEAT1 to overexpress or knockdown the NEAT1 gene. We found that overexpression of NEAT1 significantly decreased cell viability while knockdown of NEAT1 increased cell viability of HG-treated HBZY1 cells (135.721 \pm 6.098 vs. $179.213 \pm$ $6.462, p<0.01 ; 256.615 \pm 4.377$ vs. $219.745 \pm 7.462, p<0.05$; respectively) (Fig. 2 B). Meanwhile, overexpression of NEAT1 upregulated the gene expression as well as the protein levels of NLRP3, caspase-1, and IL-1 $\beta$ (Fig. 2C, D). Similarly, knockdown of NEAT1 downregulated the expressions of those proteins. Furthermore, we found that cells transfected with pcDNANEAT1 had higher percentages while cells transfected with sh-NEAT1 had lower percentages of pyroptosis when exposed to HG $(26.231 \pm 1.246$ vs. $17.562 \pm 1.752, p<0.05 ; 4.342 \pm 2.011$ vs. $17.335 \pm 0.971, p<0.01$; respectively) (Fig. $2 \mathrm{E}$ ). Taken together, these results confirm that NEAT1 promotes cell pyroptosis in DN.

\section{miR-34c Is a Target of NEAT1}

In order to elucidate the molecular mechanism of how NEAT1 contributes to the pathogenesis of DN, we searched the possible targets of NEAT1 using bioinformatics methods. miR-34c was predicted to bind to NEAT1 (the binding sequences are shown in Fig. 3A). Luciferase activity assay was then performed to confirm whether miR-34c was a direct target of NEAT1. Luciferase activity was significantly decreased in cells transfected with miR-34c mimics $(0.543 \pm 0.047$ vs. $1.003 \pm 0.120, p<0.05)$ while this effect was reversed when NEAT1 binding was mutated (Fig. 3B). qPCR also revealed that the expression of miR-34c was reduced in the presence of pcDNA-NEAT1 and increased in the presence of sh-NEAT1 compared to its expression in control cells $(0.372 \pm 0.102$ vs. $1.003 \pm 0.076, p<0.05 ; 1.864 \pm 0.142$ vs. 1.003 $\pm 0.076, p<0.01$; respectively) (Fig. $3 \mathrm{C}$ ), suggesting that miR-34c is a potential target of NEAT1 and its expression is regulated by NEAT1. 


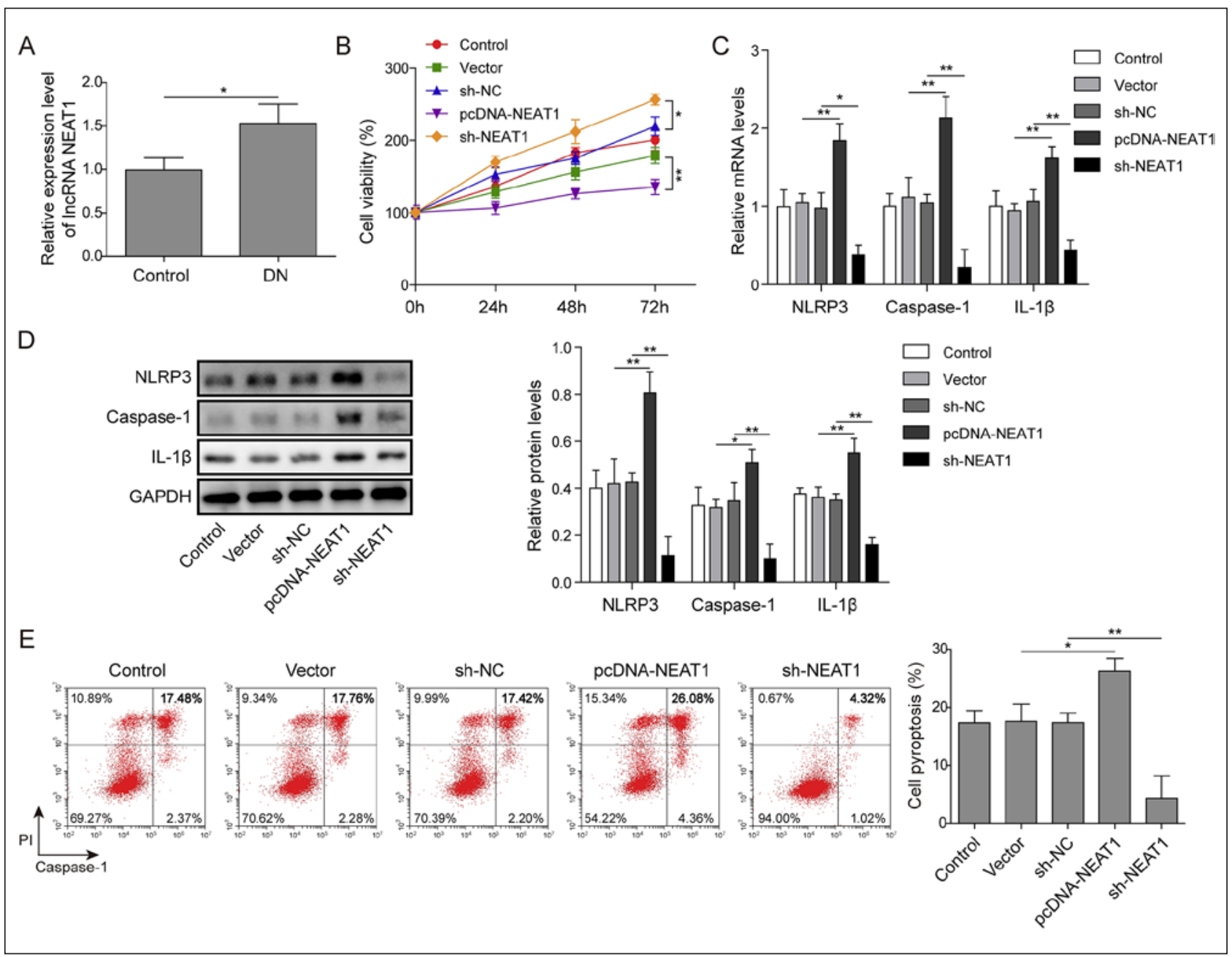

Fig. 2. NEAT1 promotes cell pyroptosis in HG-treated HBZY1 cells. A NEAT1 expression in HBZY1 cells with or without exposure to $30 \mathrm{mmol} / \mathrm{L} \mathrm{HG}$ media for $48 \mathrm{~h}$. (B-E) HBZY1 cells with or without transfection were treated with $30 \mathrm{mmol} / \mathrm{L} \mathrm{HG}$ for $48 \mathrm{~h}$ before all the measurements were taken. B Cell viability was determined by MTT assay. C mRNA expressions of NLRP3, caspase-1, and IL-1 $\beta$ by qPCR. D Western blotting determined the protein levels of NLRP3, caspase-1, and IL-1 $\beta$. E Cell pyroptosis was assayed by flow cytometry analysis. Data are expressed as means \pm SD. Bold numbers indicate mean percentages of pyroptotic cells. Comparison between groups was done using one-way ANOVA with post hoc analysis (Bonferroni test). ${ }^{*} p<0.05 ;{ }^{* *} p<0.01$.

\section{miR-34c Inhibits Cell Pyroptosis in HG-Treated HBZY1 Cells}

By using the HG-treated HBZY1 cell model, we further explored the relationship between miR-34c and pyroptosis. After achieving $70-85 \%$ confluence, the media was changed to FBS-free media to synchronize the cell growth for $24 \mathrm{~h}$. Then the cells were incubated in either serum-free media with normal glucose $(5.5 \mathrm{mmol} / \mathrm{L})$ or $\mathrm{HG}(30 \mathrm{mmol} / \mathrm{L})$ for $48 \mathrm{~h}$ before harvest. Interestingly, the gene expression of miR-34c was downregulated in HG-treated cells compared with untreated cells $(0.592 \pm 0.101$ vs. $1.012 \pm 0.101, p<0.05)$ (Fig. 4A). Moreover, cells transfected with miR-34c mimics had decreased expressions of pyroptosisrelated proteins, increased cell viability, and lower percentages of pyroptosis under hyperglycaemic conditions, which were reversed by suppression of miR-34c with miR-34c inhibitor transfection (Fig. 4B-D). Our findings suggest that miR-34c inhibits cell pyroptosis in DN. 


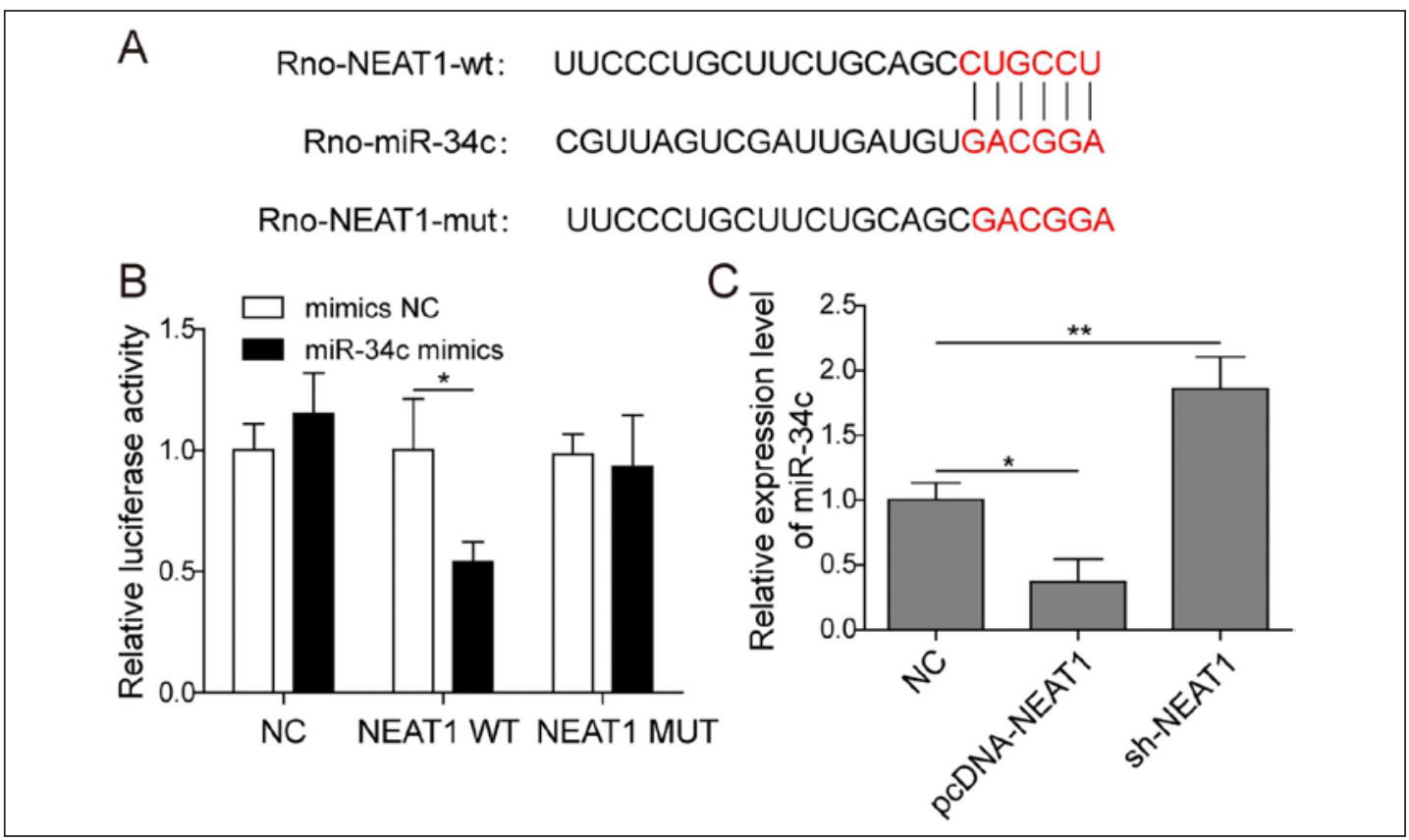

Fig. 3. NEAT1 regulates the expression of miR-34c. A Sequence complementarity between NEAT1 and miR$34 \mathrm{c}$ using bioinformatics methods. B The targeting association between NEAT1 and miR-34c by dual-luciferase reporter assay in HBZY1 cells transfected with WT-NEAT1 or MUT-NEAT1 and miR-34c or miR-NC. C The relative expressions of miR-34c by qPCR in HBZY1 cells transfected with pcDNA-NEAT1 or sh-NEAT1. Data are expressed as means $\pm \mathrm{SD}$. Comparisons were conducted using one-way ANOVA. ${ }^{*} p<0.05 ;{ }^{* *} p<0.01$.

\section{Pyroptosis-Related Protein NLPR3 Is a Target of miR-34c}

We further explored the downstream target genes of miR-34 and found that NLRP3 $3^{\prime}$-UTR contained a conserved binding site for miR-34c, and this binding site was also present in the sequence of an NLRP3 3'-UTR mutant (Fig. 5A). Luciferase reporter assays showed that miR-34c significantly decreased the luciferase activity of the wild-type $3^{\prime}$-UTR of NLRP3 $(0.303 \pm 0.102$ vs. $0.997 \pm 0.038, p<0.01)$ but not that of the mutant-type $3^{\prime}$-UTR of NLRP3, confirming that NLRP3 is a target gene of miR-34c (Fig. 5B). qPCR and Western blot showed that overexpression of miR-34c decreased the mRNA and protein expressions of NLRP3 (qPCR: $0.553 \pm 0.075$ vs. $1.001 \pm 0.131, p<0.05$; Western blot: $0.163 \pm 0.026$ vs. $0.412 \pm 0.035$, $p<0.05$ ) while inhibition of miR-34c increased the expression of NLRP3 (qPCR: $1.637 \pm 0.067$ vs. $1.001 \pm 0.131, p<0.01$; Western blot: $0.843 \pm 0.075$ vs. $0.412 \pm 0.035, p<0.01$ ) (Fig. 5 C, D), suggesting that miR-34c regulates the expression of NLRP3, which is a key mediator of pyroptosis.

\section{NEAT1 Regulates Pyroptosis and Inflammation via Targeting miR-34c/NLRP3}

We further examined whether the effects of NEAT1 in HBZY1 cells exposed to HG are associated with the regulation of miR-34c/NLRP3. HBZY1 cells were transfected with sh-NEAT1, with the combination of miR-34c inhibitor or pcDNA-NLRP3, followed by HG treatment. The gene expressions and protein levels of NLRP3, caspase-1, and IL-1 $\beta$ were markedly reduced in cells transfected with sh-NEAT1, compared with the control group, whereas co-transfection with miR-34c inhibitor or pcDNA-NLRP3 significantly increased the gene expression and protein levels of those proteins (Fig. 6A, B). Measurements of cell viability and pyroptosis also showed consistent results (Fig. 6C, D). Either miR-34c inhibition or 


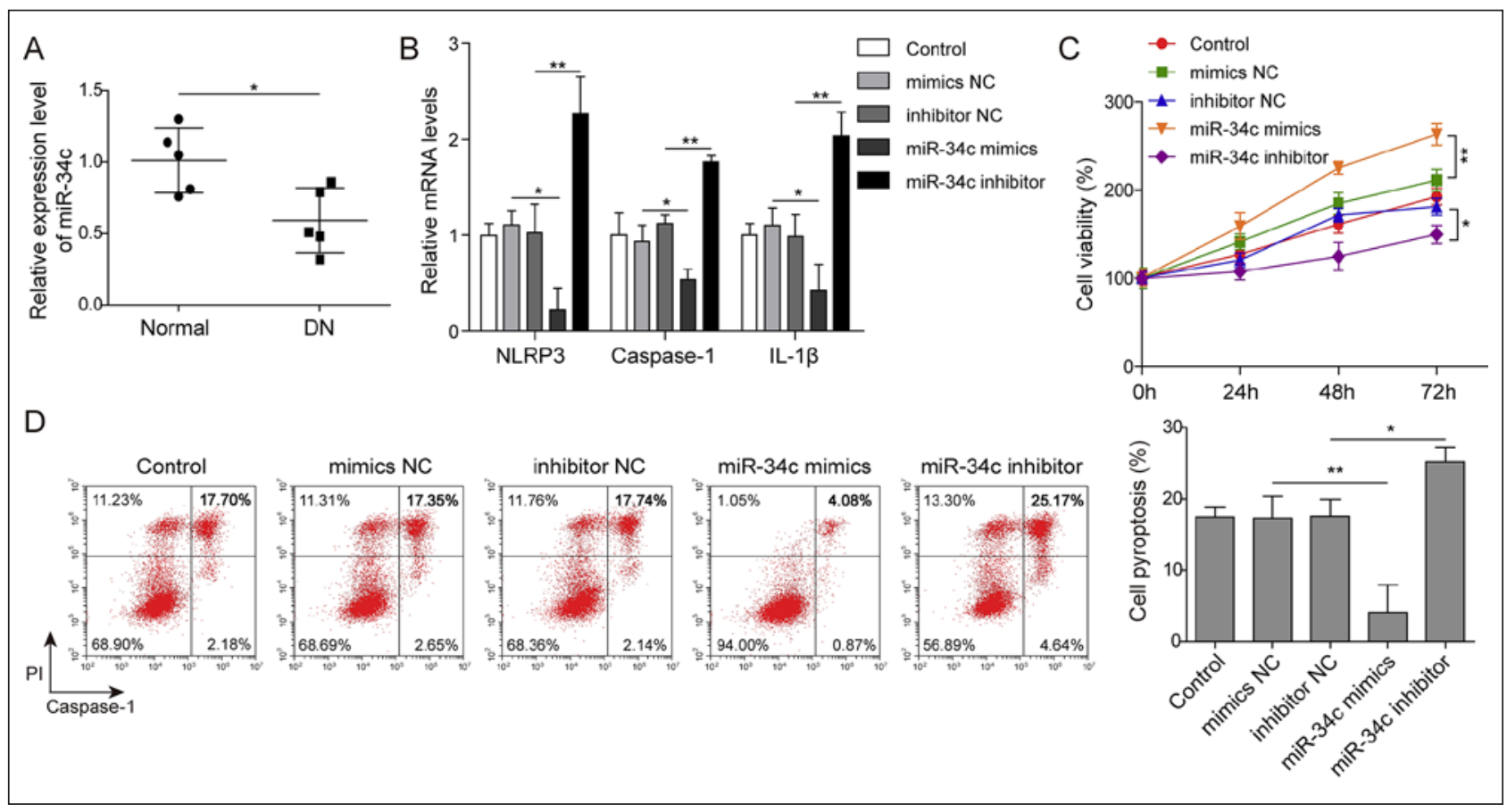

Fig. 4. miR-34c inhibits cell pyroptosis in HG-treated HBZY1 cells. A miR-34c expression in HBZY1 cells with or without exposure to HG. B-D HBZY1 cells with or without transfection were treated with HG before all the measurements were taken. B mRNA expressions of NLRP3, caspase-1, and IL-1 $\beta$ by qPCR. C Cell viability was determined by MTT assay. D Cell pyroptosis was assayed by flow cytometry analysis. Bold numbers indicate mean percentages of pyroptotic cells. Data are expressed as means \pm SD. Comparison between groups was done using one-way ANOVA with post hoc analysis (Bonferroni test). ${ }^{*} p<0.05$; ${ }^{* *} p<0.01$.

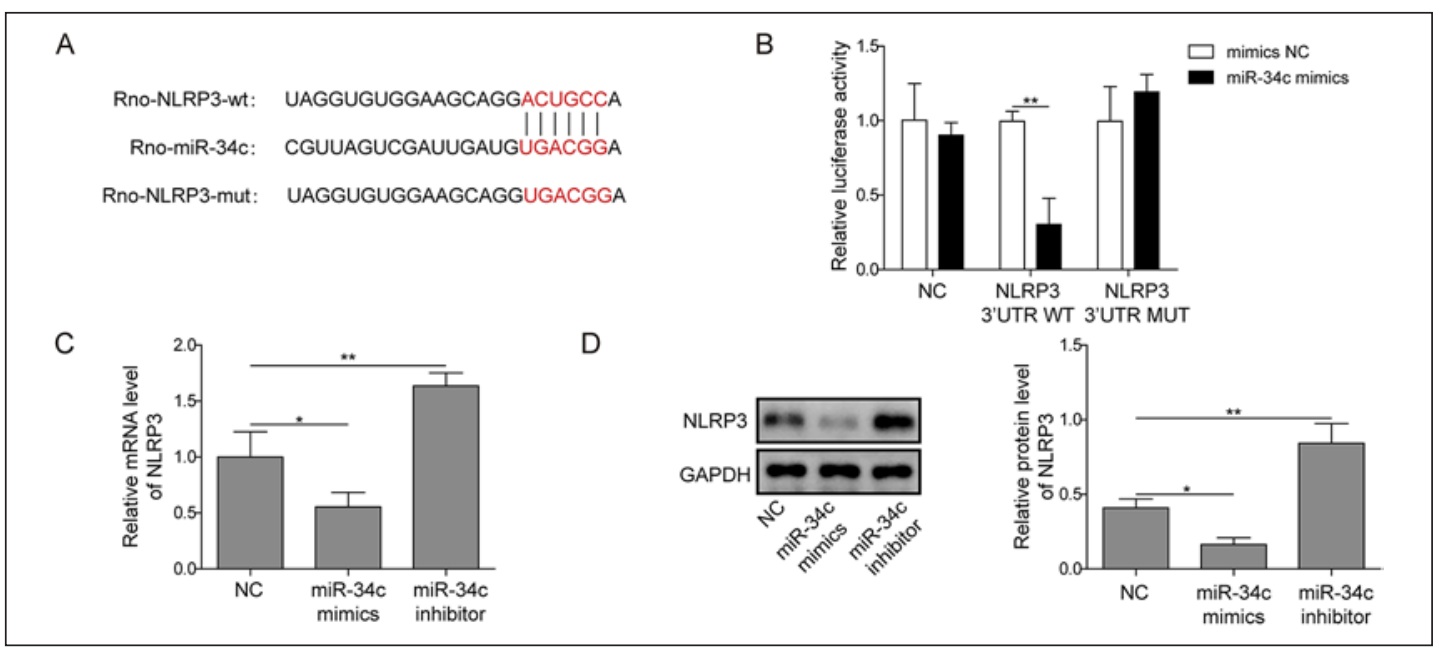

Fig. 5. miR-34c regulates the expression of NLRP3. A Sequence complementarity between miR-34c and NLRP3 using bioinformatics methods. B The targeting association between miR-34c and NLRP3 by dual-luciferase reporter assay in HBZY1 cells transfected with WT-NLRP3 or MUT-NLRP3 and miR-34c or miR-NC. C The relative expressions of NLRP3 in HBZY1 cells transfected with miR-34c mimics or miR-34c inhibitor. D The protein levels of NLRP3 by Western blot in HBZY1 cells transfected with miR-34c mimics or miR-34c inhibitor. Data are expressed as means \pm SD. Comparisons were conducted using one-way ANOVA. ${ }^{*} p<0.05$; $* * p<0.01$. 


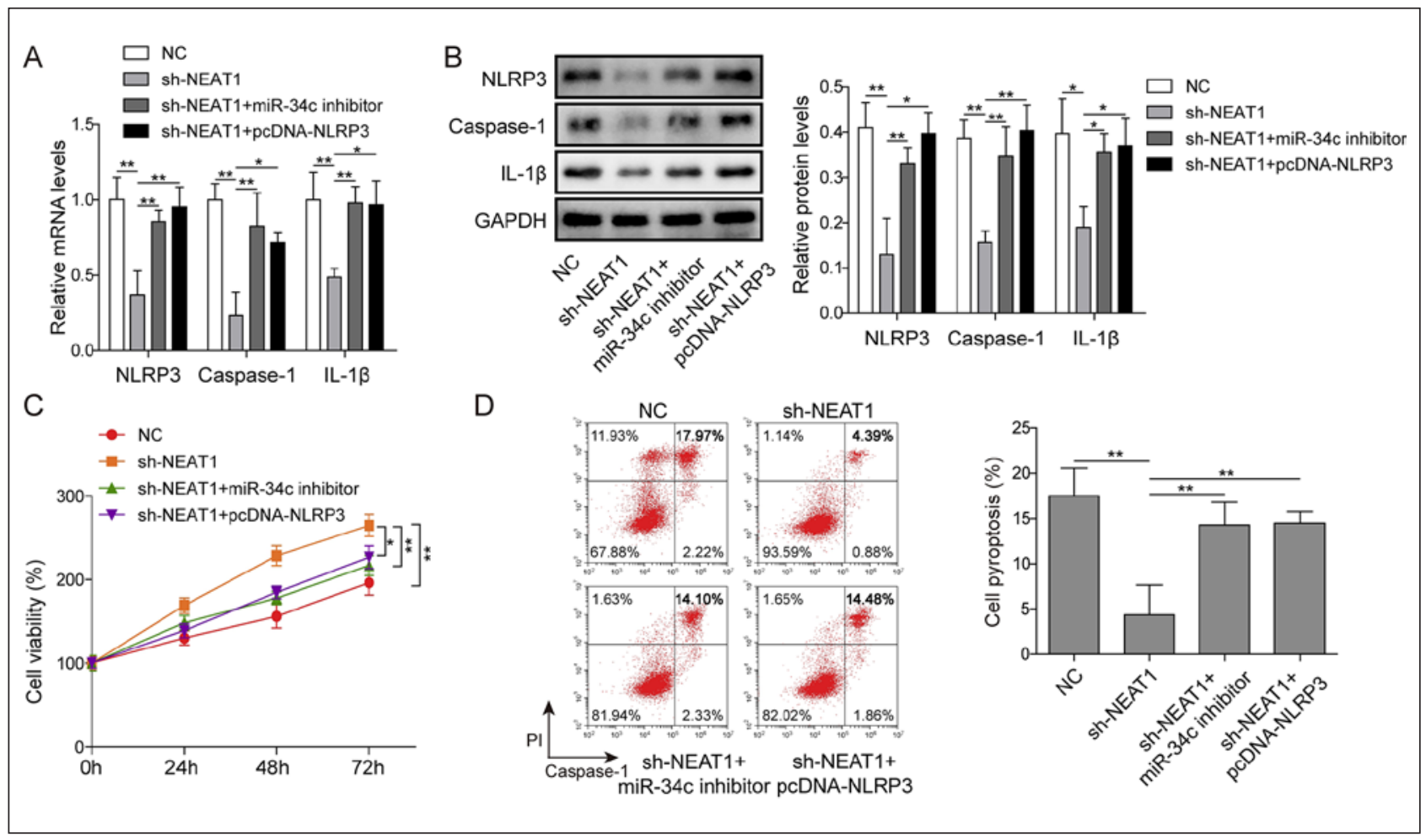

Fig. 6. NEAT1 regulates pyroptosis and inflammation via targeting miR-34c/NLRP3. HBZY1 cells were transfected with sh-NEAT1, with the combination of miR-34c inhibitor or pcDNA-NLRP3, followed by HG treatment before all the measurements were taken. A mRNA expressions of NLRP3, caspase-1, and IL-1 $\beta$ by qPCR. B Western blotting determined the protein levels of NLRP3, caspase-1, and IL-1 $\beta$. C Cell viability was determined by MTT assay. D Cell pyroptosis was assayed by flow cytometry analysis. Bold numbers indicate mean percentages of pyroptotic cells. Data are expressed as means \pm SD. Comparison between groups was done using one-way ANOVA with post hoc analysis (Bonferroni test). ${ }^{*} p<0.05$; ${ }^{* *} p<0.01$.

NLRP3 overexpression could reverse the accentuation of pyroptosis and inflammation by sh-NEAT1 transfection in the in vitro model of DN. Thus, we conclude that NEAT1 impacts cell pyroptosis and inflammation in DN via regulating miR-34c/NLRP3.

\section{Discussion}

In the present study, we examined the potential role of pyroptosis in DN pathogenesis and the possible underlying molecular mechanisms in an in vivo rat model and an in vitro glomerular cell line. We found that the pyroptosis and subsequent inflammation, regulated by the NEAT1/miR-34c/NLRP3 signalling axis, contribute to the development of DN.

DN is traditionally considered to result from the metabolic and haemodynamic alterations other than being an inflammatory disease. However, intensive research in recent years reveals that the aetiology of DN seems to be multifactorial wherein inflammatory mechanisms play a key role in the development and progression of $\mathrm{DN}$, with recruitment and activation of immune cells and elaboration of proinflammatory cytokines [28]. Indeed, the accumulation of inflammatory cells in the kidneys has been shown in both DN patients and mouse models of DN while the extent of accumulation is associated with the progress of renal 


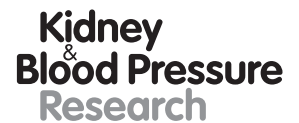

Kidney
Blood Pressure

Research \begin{tabular}{l|l}
\hline Kidney Blood Press Res 2020;45:589-602 \\
\hline DOI: 10.1159/000508372 & $\begin{array}{l}\text { @ 2020 The Author(s). Published by S. Karger AG, Basel } \\
\text { www.karger.com/kbr }\end{array}$ \\
\hline
\end{tabular}

Zhan et al.: IncRNA NEAT1/miR-34c/NLRP3 Axis Regulates Pyroptosis in DN

dysfunction [29-31]. Moreover, local and circulating levels of proinflammatory cytokines are increased in humans and animals with DN $[32,33]$. However, the cause of inflammation in DN is still unclear. The pathogens and infections associated with diabetes as well as the cell injury/damage caused by the hyperglycaemic condition may be responsible. Pyroptosis, a highly inflammatory form of programmed cell death, is believed to be triggered by intracellular pathogens and/or cell damage [34], suggesting a possible link between pyroptosis and the inflammation associated with DN. As the result of inflammasome-mediated caspase-1 activation, pyroptosis is also suggested to be another important contributor to DN supported by previous observations, which is consistent with the well-supported concept of sterile inflammation in DN [3]. For example, NLRP-3 inflammasome activation has been previously documented to aggravate DN in diabetic mice [35]. Moreover, it has been shown that deficiency of caspase- 1 supressed inflammasome activation and protected against DN in mice, suggesting that pyroptosis has a crucial function in DN [5]. In the present study, we found increased levels of pyroptosis-related proteins and proinflammatory cytokines as well as increased pyroptotic cell percentage in an in vivo rat model of DN, providing further evidence for the involvement of pyroptosis in the pathogenesis of DN.

A number of studies have suggested the relationship between lncRNAs/miRNAs and pyroptosis. Several lncRNAs, such as MALAT1 and KCNQ10T1, and miRNAs, such as miR214-3p, miR-23c, and miR-22-3p, have been identified to be involved in the cell pyroptosis mechanism in diabetes-related nephropathy, cardiomyopathy, and atherosclerosis [36-38]. Consistent with our observations, NEAT1 was significantly increased in STZ-induced DN rat models and HG-induced rat mesangial cells; the function of NEAT1 in DN was proposed to promote fibrogenesis $[13,14]$. On the other hand, NEAT1 has been demonstrated to enhance activation of inflammasomes and subsequent caspase-1-dependent pyroptosis and reduce inflammatory responses in mouse macrophages [15]. The role of NEAT1 in DN-related pyroptosis is therefore suspected. In the present study, we found that NEAT1 promoted pyroptosis in DN via regulating miR-34c, suggesting a significant role of NEAT1 in the pathogenesis of DN.

Several miRNAs are involved in the pathogenesis of DN. miR-34c has been found to inhibit fibrosis, apoptosis, and EMT in the kidney [20]. However, the role of miR-34c in DN has not been well documented. miRNA is a key regulator of cellular process including pyroptosis, although only limited miRNAs have been found to be meaningful mediators of pyroptosis [39]. We identified that the pyroptosis-related protein NLPR3 was a target of miR-34c in DN, implicating the regulating role of miR-34c in pyroptosis.

The NLRP family, consisting of more than 13 members, is the critical participant in the formation of inflammasomes and initiation of the immune response, and has been known to be associated with a number of diseases. NLRP3 inflammasome is the most studied inflammasome and has been proposed to be a potential therapeutic target for the treatment of DN supported by increasing evidence [40,41]. Several studies have also shown promising results for DN treatment by targeting NLRP3 [42-44]. NLRP3, along with the adaptor protein apoptosis-associated spec-like protein containing a CARD (ASC), activates caspase- 1 through inflammasome assembly and mediates the change of IL-1 $\beta$ from an inactive precursor to the secreted active form and the process of pyroptosis [45]. We found that miR-34c regulated the expression of NLRP3 as well as the expressions of caspase- 1 and IL-1 $\beta$ in DN, suggesting the role of the NLRP3/caspase-1/IL-1 $\beta$ axis in DN. In addition, a recent study by Wu et al. [46] demonstrated that NLRP3 knockout reduced oxidative stress, attenuated renal inflammation and fibrosis, as well as improved renal function in STZ-induced diabetic mice. In the present study, we found that NEAT1/miR-34c regulated the expression of NLRP3 and cell pyroptosis and revealed a potential regulating pathway of pyroptosis in DN.

In sum, our study demonstrated that NEAT1 and its target gene miR-34c regulated cell pyroptosis via mediating NLRP3 in DN, providing new insights into understanding 
the molecular mechanisms of pyroptosis in the pathogenesis of DN and presenting further evidence for the therapeutic potentials for DN by targeting pyroptosis-related pathways.

\section{Statement of Ethics}

This animal study was performed in accordance with the guidelines of the National Institutes of Health Guide for the Care and Use of Laboratory Animals. All protocols were approved by the Animal Ethics Committee of the Second Affiliated Hospital of Nanchang University.

\section{Conflict of Interest Statement}

The authors declare that they have no conflict of interest.

\section{Funding Sources}

the authors have no funding sources to declare.

\section{Author Contributions}

Guarantor of integrity of the entire study: Wen-Wei Xu. Study concepts and design: Jin-Feng Zhan and Wen-Wei Xu. Definition of intellectual content: Jin-Feng Zhan, Wen-Wei Xu, and Hong-Wei Huang. Literature research: Jin-Feng Zhan, Wen-Wei Xu, Hong-Wei Huang, and Chong Huang. Clinical studies: Jin-Feng Zhan, Hong-Wei Huang, and Li-Li Hu. Experimental studies: Jin-Feng Zhan, Hong-Wei Huang, and Chong Huang. Data acquisition and analysis: Jin-Feng Zhan, Hong-Wei Huang, Chong Huang, and Li-Li Hu. Statistical analysis: Jin-Feng Zhan, Wen-Wei Xu, and Hong-Wei Huang. Manuscript preparation: Jin-Feng Zhan, Hong-Wei Huang, Chong Huang, and Li-Li Hu. Manuscript editing: Jin-Feng Zhan, Hong-Wei Huang, and Li-Li Hu. Manuscript review: Jin-Feng Zhan and Wen-Wei Xu.

\section{References}

1 Najafian B, Fogo AB, Lusco MA, Alpers CE. AJKD Atlas of Renal Pathology: diabetic nephropathy. Am J Kidney Dis. 2015 Nov;66(5):e37-8.

2 Magee C, Grieve DJ, Watson CJ, Brazil DP. Diabetic Nephropathy: a Tangled Web to Unweave. Cardiovasc Drugs Ther. 2017 Dec;31(5-6):579-92.

3 Tesch GH. Diabetic nephropathy - is this an immune disorder? Clin Sci (Lond). 2017 Jul;131(16):2183-99.

4 Jiang D, Chen S, Sun R, Zhang X, Wang D. The NLRP3 inflammasome: role in metabolic disorders and regulation by metabolic pathways. Cancer Lett. 2018 Apr;419:8-19.

5 Shahzad K, Bock F, Al-Dabet MM, Gadi I, Kohli S, Nazir S, et al. Caspase-1, but Not Caspase-3, Promotes Diabetic Nephropathy. J Am Soc Nephrol. 2016 Aug;27(8):2270-5.

6 Bălăşescu E, Ion DA, Cioplea M, Zurac S. Caspases, Cell Death and Diabetic Nephropathy. Rom J Intern Med. 2015 Oct-Dec;53(4):296-303.

7 Bergsbaken T, Fink SL, Cookson BT. Pyroptosis: host cell death and inflammation. Nat Rev Microbiol. 2009 Feb;7(2):99-109.

8 Schmitz SU, Grote P, Herrmann BG. Mechanisms of long noncoding RNA function in development and disease. Cell Mol Life Sci. 2016 Jul;73(13):2491-509.

9 Li Y, Xu K, Xu K, Chen S, Cao Y, Zhan H. Roles of Identified Long Noncoding RNA in Diabetic Nephropathy. J Diabetes Res. 2019 Feb;2019:5383010. 
10 Duan LJ, Ding M, Hou LJ, Cui YT, Li CJ, Yu DM. Long noncoding RNA TUG1 alleviates extracellular matrix accumulation via mediating microRNA-377 targeting of PPAR $\gamma$ in diabetic nephropathy. Biochem Biophys Res Commun. 2017 Mar;484(3):598-604.

11 Long J, Badal SS, Ye Z, Wang Y, Ayanga BA, Galvan DL, et al. Long noncoding RNA Tug1 regulates mitochondrial bioenergetics in diabetic nephropathy. J Clin Invest. 2016 Nov;126(11):4205-18.

12 Gao J, Wang W, Wang F, Guo C. LncRNA-NR_033515 promotes proliferation, fibrogenesis and epithelial-tomesenchymal transition by targeting miR-743b-5p in diabetic nephropathy. Biomed Pharmacother. 2018 Oct; 106:543-52.

13 Huang S, Xu Y, Ge X, Xu B, Peng W, Jiang X, et al. Long noncoding RNA NEAT1 accelerates the proliferation and fibrosis in diabetic nephropathy through activating Akt/mTOR signaling pathway. J Cell Physiol. 2019 Jul; 234(7):11200-7.

14 Wang X, Xu Y, Zhu YC, Wang YK, Li J, Li XY, et al. LncRNA NEAT1 promotes extracellular matrix accumulation and epithelial-to-mesenchymal transition by targeting miR-27b-3p and ZEB1 in diabetic nephropathy. J Cell Physiol. 2019 Aug;234(8):12926-33.

15 Zhang P, Cao L, Zhou R, Yang X, Wu M. The lncRNA Neat1 promotes activation of inflammasomes in macrophages. Nat Commun. 2019 Apr;10(1):1495.

16 Zhang Y, Sun X, Icli B, Feinberg MW. Emerging Roles for MicroRNAs in Diabetic Microvascular Disease: Novel Targets for Therapy. Endocr Rev. 2017 Apr;38(2):145-68.

17 Zhao Y, Yin Z, Li H, Fan J, Yang S, Chen C, et al. MiR-30c protects diabetic nephropathy by suppressing epithelialto-mesenchymal transition in db/db mice. Aging Cell. 2017 Apr;16(2):387-400.

18 Guo J, Li J, Zhao J, Yang S, Wang L, Cheng G, et al. MiRNA-29c regulates the expression of inflammatory cytokines in diabetic nephropathy by targeting tristetraprolin. Sci Rep. 2017 May;7(1):2314.

19 Gholaminejad A, Abdul Tehrani H, Gholami Fesharaki M. Identification of candidate microRNA biomarkers in diabetic nephropathy: a meta-analysis of profiling studies. J Nephrol. 2018 Dec;31(6):813-31.

20 Liu XD, Zhang LY, Zhu TC, Zhang RF, Wang SL, Bao Y. Overexpression of miR-34c inhibits high glucose-induced apoptosis in podocytes by targeting Notch signaling pathways. Int J Clin Exp Pathol. 2015 May;8(5):4525-34.

21 Xu L, Wang Q, Jiang W, Yu S, Zhang S. MiR-34c Ameliorates Neuropathic Pain by Targeting NLRP3 in a Mouse Model of Chronic Constriction Injury. Neuroscience. 2019 Feb;399:125-34.

22 Tesch GH, Allen TJ. Rodent models of streptozotocin-induced diabetic nephropathy. Nephrology (Carlton). 2007 Jun;12(3):261-6.

23 Xu ZG, Li SL, Lanting L, Kim YS, Shanmugam N, Reddy MA, et al. Relationship between 12/15-lipoxygenase and COX-2 in mesangial cells: potential role in diabetic nephropathy. Kidney Int. 2006 Feb;69(3):512-9.

24 Liu F, Ma XJ, Wang QZ, Zhao YY, Wu LN, Qin GJ. The effect of FoxO1 on the proliferation of rat mesangial cells under high glucose conditions. Nephrol Dial Transplant. 2014 Oct;29(10):1879-87.

25 Han F, Xue M, Chang Y, Li X, Yang Y, Sun B, et al. Triptolide Suppresses Glomerular Mesangial Cell Proliferation in Diabetic Nephropathy Is Associated with Inhibition of PDK1/Akt/mTOR Pathway. Int J Biol Sci. 2017 Sep; 13(10):1266-75.

26 Bustin SA, Benes V, Garson JA, Hellemans J, Huggett J, Kubista M, et al. The MIQE guidelines: minimum information for publication of quantitative real-time PCR experiments. Clin Chem. 2009 Apr;55(4):611-22.

27 Schmittgen TD, Livak KJ. Analyzing real-time PCR data by the comparative C(T) method. Nat Protoc. 2008; 3(6):1101-8.

28 Lim AK, Tesch GH. Inflammation in diabetic nephropathy. Mediators Inflamm. 2012;2012:146154.

29 Chow F, Ozols E, Nikolic-Paterson DJ, Atkins RC, Tesch GH. Macrophages in mouse type 2 diabetic nephropathy: correlation with diabetic state and progressive renal injury. Kidney Int. 2004 Jan;65(1):116-28.

30 Chow FY, Nikolic-Paterson DJ, Atkins RC, Tesch GH. Macrophages in streptozotocin-induced diabetic nephropathy: potential role in renal fibrosis. Nephrol Dial Transplant. 2004 Dec;19(12):2987-96.

31 Nguyen D, Ping F, Mu W, Hill P, Atkins RC, Chadban SJ. Macrophage accumulation in human progressive diabetic nephropathy. Nephrology (Carlton). 2006 Jun;11(3):226-31.

32 Navarro JF, Milena FJ, Mora C, León C, García J. Renal pro-inflammatory cytokine gene expression in diabetic nephropathy: effect of angiotensin-converting enzyme inhibition and pentoxifylline administration. Am J Nephrol. 2006;26(6):562-70.

33 Uzun S, Ozari M, Gursu M, Karadag S, Behlul A, Sari S, et al. Changes in the inflammatory markers with advancing stages of diabetic nephropathy and the role of pentraxin-3. Ren Fail. 2016 Sep;38(8):1193-8.

34 Vande Walle L, Lamkanfi M. Pyroptosis. Curr Biol. 2016 Jul;26(13):R568-72.

35 Shahzad K, Bock F, Dong W, Wang H, Kopf S, Kohli S, et al. Nlrp3-inflammasome activation in non-myeloidderived cells aggravates diabetic nephropathy. Kidney Int. 2015 Jan;87(1):74-84.

36 Yang F, Qin Y, Wang Y, Li A, Lv J, Sun X, et al. LncRNA KCNQ10T1 Mediates Pyroptosis in Diabetic Cardiomyopathy. Cell Physiol Biochem. 2018;50(4):1230-44.

37 Song Y, Yang L, Guo R, Lu N, Shi Y, Wang X. Long noncoding RNA MALAT1 promotes high glucose-induced human endothelial cells pyroptosis by affecting NLRP3 expression through competitively binding miR-22. Biochem Biophys Res Commun. 2019 Feb;509(2):359-66.

38 Li X, Zeng L, Cao C, Lu C, Lian W, Han J, et al. Long noncoding RNA MALAT1 regulates renal tubular epithelial pyroptosis by modulated miR-23c targeting of ELAVL1 in diabetic nephropathy. Exp Cell Res. 2017 Jan; 350(2):327-35. 
39 Lee S, Choi E, Cha MJ, Hwang KC. Looking for Pyroptosis-Modulating miRNAs as a Therapeutic Target for Improving Myocardium Survival. Mediators Inflamm. 2015;2015:254871.

40 Fu Y, Wu N, Zhao D. Function of NLRP3 in the Pathogenesis and Development of Diabetic Nephropathy. Med Sci Monit. 2017 Aug;23:3878-84.

41 Qiu YY, Tang LQ. Roles of the NLRP3 inflammasome in the pathogenesis of diabetic nephropathy. Pharmacol Res. 2016 Dec;114:251-64.

42 Xin R, Sun X, Wang Z, Yuan W, Jiang W, Wang L, et al. Apocynin inhibited NLRP3/XIAP signalling to alleviate renal fibrotic injury in rat diabetic nephropathy. Biomed Pharmacother. 2018 Oct;106:1325-31.

43 Song W, Wei L, Du Y, Wang Y, Jiang S. Protective effect of ginsenoside metabolite compound K against diabetic nephropathy by inhibiting NLRP3 inflammasome activation and NF- $\kappa B / p 38$ signaling pathway in high-fat diet/streptozotocin-induced diabetic mice. Int Immunopharmacol. 2018 Oct;63:227-38.

44 Wang S, Li Y, Fan J, Zhang X, Luan J, Bian Q, et al. Interleukin-22 ameliorated renal injury and fibrosis in diabetic nephropathy through inhibition of NLRP3 inflammasome activation. Cell Death Dis. 2017 Jul;8(7):e2937.

45 Strowig T, Henao-Mejia J, Elinav E, Flavell R. Inflammasomes in health and disease. Nature. 2012 Jan; 481(7381):278-86.

46 Wu M, Han W, Song S, Du Y, Liu C, Chen N, et al. NLRP3 deficiency ameliorates renal inflammation and fibrosis in diabetic mice. Mol Cell Endocrinol. 2018 Dec;478:115-25. 